\title{
The Discrete Case of the Mixed Joint Universality for a Class of Certain Partial Zeta-functions
}

\author{
Roma Kačinskaitè and Kohji Matsumoto*
}

\begin{abstract}
We give a new type of mixed discrete joint universality properties, which is satisfied by a wide class of zeta-functions. We study the universality for a certain modification of Matsumoto zeta-functions $\varphi_{h}(s)$ and a collection of periodic Hurwitz zeta-functions $\zeta(s, \alpha ; \mathfrak{B})$ under the condition that the common difference of arithmetical progression $h>0$ is such that $\exp \left\{\frac{2 \pi}{h}\right\}$ is a rational number and parameter $\alpha$ is a transcendental number.
\end{abstract}

\section{Introduction}

In 2015, the first result on the mixed joint universality theorem for a general polynomial Euler product (or a so-called Matsumoto zeta-function) $\varphi(s)$ belonging to the Steuding class $\widetilde{S}$ and a periodic Hurwitz zeta-function $\zeta(s, \alpha ; \mathfrak{B})$ was obtained by the authors (see [5]). In 2017, this result was generalized to the case of the tuple consisting of one Matsumoto zeta-function and several periodic Hurwitz zeta-functions (see [7]).

We recall the definitions of both of the above functions. Let $s=\sigma+i t$ be a complex variable, and by $\mathbb{P}, \mathbb{N}, \mathbb{N}_{0}, \mathbb{Z}, \mathbb{Q}$ and $\mathbb{C}$ denote the sets of all primes, positive integers, non-negative integers, integers, rational numbers and complex numbers, respectively. Let $\mathfrak{B}=\left\{b_{m}: m \in \mathbb{N}_{0}\right\}$ be a periodic sequence of complex numbers $b_{m}$ with a minimal period $k \in \mathbb{N}$, and suppose that $\alpha$ is a fixed real number, $0<\alpha \leq 1$. Then, for $\sigma>1$, the periodic Hurwitz zeta-function is defined by the Dirichet series

$$
\zeta(s, \alpha ; \mathfrak{B})=\sum_{m=0}^{\infty} \frac{b_{m}}{(m+\alpha)^{s}} .
$$

For $\sigma>1$, the function $\zeta(s, \alpha ; \mathfrak{B})$ can be expressed as a linear combination of classical Hurwitz zeta-functions $\zeta(s, \alpha)$ (see $[10]$ ), i.e.,

$$
\zeta(s, \alpha ; \mathfrak{B})=\frac{1}{k^{s}} \sum_{l=0}^{k-1} b_{l} \zeta\left(s, \frac{l+\alpha}{k}\right),
$$

Received July 15, 2020; Accepted February 1, 2021.

Communicated by Ming-Lun Hsieh.

2020 Mathematics Subject Classification. 11M06, 11M41, 11M36, 41A30, 30E10.

Key words and phrases. approximation, discrete shift, Euler products, periodic Hurwitz zeta-function, Matsumoto zeta-function, value distribution, universality.

*Corresponding author. 
from which we deduce that it can be analytically continued to the whole $s$-plane except for a possible simple pole at the point $s=1$ with residue $b:=k^{-1}\left(b_{0}+\cdots+b_{k-1}\right)$.

The polynomial Euler products $\widetilde{\varphi}(s)$ or so-called Matsumoto zeta-functions are given by the formula

$$
\widetilde{\varphi}(s)=\prod_{m=1}^{\infty} \prod_{j=1}^{g(m)}\left(1-\frac{a_{m}^{(j)}}{p_{m}^{s f(j, m)}}\right)^{-1}
$$

for $m \in \mathbb{N}, g(m) \in \mathbb{N}, j \in \mathbb{N}, 1 \leq j \leq g(m), f(j, m) \in \mathbb{N}$, and the $m$ th prime number $p_{m}$ (see [14]). Suppose that, for non-negative constants $\alpha$ and $\beta$, the inequalities

$$
g(m) \leq C_{1} p_{m}^{\alpha} \quad \text { and } \quad\left|a_{m}^{(j)}\right| \leq p_{m}^{\beta}
$$

hold with a positive constant $C_{1}$. In view of this assumption, the right-side of the equality (1.1) converges absolutely for $\sigma>\alpha+\beta+1$, and in this half-plane the function $\widetilde{\varphi}(s)$ can be presented by the Dirichlet series

$$
\widetilde{\varphi}(s)=\sum_{k=1}^{\infty} \frac{\widetilde{c}_{k}}{k^{s}}
$$

where the coefficients $\widetilde{c}_{k}$ satisfy an estimate $\widetilde{c}_{k}=O\left(k^{\alpha+\beta+\varepsilon}\right)$ with every positive $\varepsilon$ if all prime factors of $k$ are large (for the comments, see Appendix in [7]). For brevity, denote the shifted version of $\widetilde{\varphi}(s)$ by

$$
\varphi(s):=\widetilde{\varphi}(s+\alpha+\beta)=\sum_{k=1}^{\infty} \frac{c_{k}}{k^{s}},
$$

where $c_{k}:=k^{-\alpha-\beta} \widetilde{c}_{k}$. Then $\varphi(s)$ is an absolutely convergent series for $\sigma>1$. Also, let the function $\varphi(s)$ be such that

(i) it can be continued meromorphically to $\sigma \geq \sigma_{0}, 1 / 2 \leq \sigma_{0}<1$, and all poles in this region are included in a compact set which has no intersection with the line $\sigma=\sigma_{0}$,

(ii) for $\sigma \geq \sigma_{0}, \varphi(\sigma+i t)=O\left(|t|^{C_{2}}\right)$ holds with a positive constant $C_{2}$,

(iii) it holds the mean-value estimate

$$
\int_{0}^{T}\left|\varphi\left(\sigma_{0}+i t\right)\right|^{2} \mathrm{~d} t=O(T), \quad T \rightarrow \infty .
$$

We denote the set of all such $\varphi(s)$ by $\mathcal{M}$.

Now we recall the definition of the Steuding class $\widetilde{S}$ (see 20$]$ ). We say that the function $\varphi(s)$ belongs to this class if the following conditions are fulfilled: 
(a) there exists a Dirichlet series expansion

$$
\varphi(s)=\sum_{m=1}^{\infty} \frac{a(m)}{m^{s}}
$$

with $a(m)=O\left(m^{\varepsilon}\right)$ for every $\varepsilon>0$;

(b) there exists $\sigma_{\varphi}<1$ such that $\varphi(s)$ can be meromorphically continued to the halfplane $\sigma>\sigma_{\varphi}$, and is holomorphic except for a pole at $s=1$;

(c) for every fixed $\sigma>\sigma_{\varphi}$ and $\varepsilon>0$, there exists a constant $C_{3} \geq 0$ such that

$$
\varphi(\sigma+i t)=O\left(|t|^{C_{3}+\varepsilon}\right)
$$

(d) there exists the Euler product expansion over primes, i.e.,

$$
\varphi(s)=\prod_{p \in \mathbb{P}} \prod_{j=1}^{l}\left(1-\frac{a_{j}(p)}{p^{s}}\right)^{-1} ;
$$

(e) there exists a constant $\kappa>0$ such that

$$
\lim _{x \rightarrow \infty} \frac{1}{\pi(x)} \sum_{p \leq x}|a(p)|^{2}=\kappa,
$$

where $\pi(x)$ denotes the number of primes $p$ not exceeding $x$ as usual.

Denote by $\sigma^{*}$ the infimum of all $\sigma_{1}$ such that

$$
\frac{1}{2 T} \int_{-T}^{T}|\varphi(\sigma+i t)|^{2} \mathrm{~d} t \sim \sum_{m=1}^{\infty} \frac{|a(m)|^{2}}{m^{2 \sigma}}
$$

holds for any $\sigma \geq \sigma_{1}$. Then $1 / 2 \leq \sigma^{*}<1$. This implies that $\widetilde{S} \subset \mathcal{M}$.

Also, throughout this paper we will use the following notation and definitions. By $H(G)$ we denote the space of holomorphic functions on a region $G$ with the uniform convergence topology (here $G$ is any open region in the complex plane). Let $K \subset \mathbb{C}$ be a compact set. Denote by $H^{c}(K)$ the set of all $\mathbb{C}$-valued continuous functions on $K$ and holomorphic in the interior of $K$, and by $H_{0}^{c}(K)$ the subset of elements of $H^{c}(K)$ which are non-zero on $K$, respectively. Let $D(a, b)=\{s \in \mathbb{C}: a<\sigma<b\}$ for every $a<b$, and denote by meas $\{A\}$ the Lebesgue measure of the measurable set $A \subset \mathbb{R}$, and by $\mathcal{B}(S)$ the set of all Borel subsets of a topological space $S$.

Now we recall the statement of our first result on the mixed joint universality property, which is of continuous character, for the functions $\varphi(s)$ and $\zeta(s, \alpha ; \mathfrak{B})$. This result is Theorem 2.2 in [5] (while a more general case is contained in Theorem 4.2 in [7]). 
Theorem 1.1. (see [5]) Suppose that $\varphi(s)$ belongs to the Steuding class $\widetilde{S}$, and $\alpha$ is a transcendental number, $0<\alpha<1$. Let $K_{1}$ be a compact subset of $D\left(\sigma^{*}, 1\right), K_{2}$ be a compact subset of $D(1 / 2,1)$, both with connected complements. Then, for any $f_{1}(s) \in$ $H_{0}^{c}\left(K_{1}\right), f_{2}(s) \in H^{c}\left(K_{2}\right)$ and every $\varepsilon>0$, it holds that

$$
\begin{aligned}
\liminf _{T \rightarrow \infty} \frac{1}{T} \operatorname{meas}\{\tau \in[0, T]: & \sup _{s \in K_{1}}\left|\varphi(s+i \tau)-f_{1}(s)\right|<\varepsilon \\
& \left.\sup _{s \in K_{2}}\left|\zeta(s+i \tau, \alpha ; \mathfrak{B})-f_{2}(s)\right|<\varepsilon\right\}>0 .
\end{aligned}
$$

This theorem shows that the set of shifts $\tau$, with which the pair $(\varphi(s+i \tau), \zeta(s+$ $i \tau, \alpha ; \mathfrak{B}))$ approximates the tuple of holomorphic functions $\left(f_{1}(s), f_{2}(s)\right)$, is sufficiently rich and has a positive lower density.

The mixed joint universality property of discrete character is sometimes more interesting. In this case, we study an approximation of the functions when the imaginary part of the complex variable $s$ varies only on the values from a certain arithmetic progression with a common difference $h>0$.

In 2017 , the discrete mixed joint universality for the pair $(\varphi(s), \zeta(s, \alpha ; \mathfrak{B}))$ was proved by the authors (see [6]) under a condition for the set

$$
L(\mathbb{P}, \alpha, h):=\left\{(\log p: p \in \mathbb{P}),\left(\log (m+\alpha): m \in \mathbb{N}_{0}\right), \frac{2 \pi}{h}\right\} .
$$

Theorem 1.2. (see [6]) Let $\varphi(s), K_{1}, K_{2}, f_{1}(s)$ and $f_{2}(s)$ be as in Theorem 1.1. Suppose that the elements of the set $L(\mathbb{P}, \alpha, h)$ are linearly independent over $\mathbb{Q}$. Then, for every $\varepsilon>0$, it holds that

$$
\begin{aligned}
\liminf _{N \rightarrow \infty} \frac{1}{N+1} \#\{0 \leq k \leq N: & \sup _{s \in K_{1}}\left|\varphi(s+i k h)-f_{1}(s)\right|<\varepsilon, \\
& \left.\sup _{s \in K_{2}}\left|\zeta(s+i k h, \alpha ; \mathfrak{B})-f_{2}(s)\right|<\varepsilon\right\}>0 .
\end{aligned}
$$

Here, and in what follows, $N$ stands for a positive integer.

\section{Statements of results}

The main aim of this paper is to give a new type of mixed discrete joint universality theorem for the aforementioned functions under a different condition from that in Theorem 1.2. Suppose that $\exp \left\{\frac{2 \pi}{h}\right\} \in \mathbb{Q}$. Then we can write $\exp \left\{\frac{2 \pi}{h}\right\}=\frac{a}{b}$ for $(a, b)=1$, $a, b \in \mathbb{Z}$. Write the factorization of numbers $a$ and $b$ into primes as $a=q_{1}^{\alpha_{1}} \cdots q_{d(1)}^{\alpha_{d(1)}}$ and $b=r_{1}^{\beta_{1}} \cdots r_{d(2)}^{\beta_{d(2)}}$, respectively. Put $\mathbb{P}_{p}=\left\{q_{1}, \ldots, q_{d(1)}, r_{1}, \ldots, r_{d(2)}\right\}$, and let $\mathbb{P}_{h}:=\mathbb{P} \backslash \mathbb{P}_{p}$. 
Denote the set of all $m \in \mathbb{N}$ such that $p_{m} \in \mathbb{P}_{p}$ by $\mathbb{N}_{p}$, and let $\mathbb{N}_{h}:=\mathbb{N} \backslash \mathbb{N}_{p}$. Also by $\mathbb{N}_{m}$ denote the set of all $m \in \mathbb{N}$ whose all prime divisors belong to $\mathbb{P}_{h}$.

Under the above notation, for $\sigma>\alpha+\beta+1$, we define a modification of the Matsumoto zeta-function $\widetilde{\varphi}(s)$ by the formula

$$
\widetilde{\varphi}_{h}(s)=\prod_{m \in \mathbb{N}_{h}} \prod_{j=1}^{g(m)}\left(1-\frac{a_{m}^{(j)}}{p_{m}^{s f(j, m)}}\right)^{-1}
$$

and by $\varphi_{h}(s)$ we denote its shifted version, i.e., $\varphi_{h}(s):=\widetilde{\varphi}_{h}(s+\alpha+\beta)$. We call $\widetilde{\varphi}_{h}(s)$ and $\varphi_{h}(s)$ the partial Matsumoto zeta-functions. Note that the difference between $\varphi_{h}(s)$ and $\varphi(s)$ is only finitely many Euler factors. Therefore the function $\varphi_{h}(s)$ satisfies the properties (i), (ii) and (iii) too, so $\varphi_{h}(s) \in \mathcal{M}$. Moreover, if $\varphi(s) \in \widetilde{S}$, then $\varphi_{h}(s) \in \widetilde{S}$.

Now we are ready to give the statement of the main results in this paper. The statement of the following theorem was announced in 6].

Theorem 2.1. Suppose that $\alpha$ is transcendental, $h>0$, and $\exp \left\{\frac{2 \pi}{h}\right\}$ is a rational number. Let $\varphi_{h}(s) \in \widetilde{S}$. Suppose $K_{1}, K_{2}, f_{1}(s)$ and $f_{2}(s)$ satisfy the conditions of Theorem 1.1 . Then, for every $\varepsilon>0$, it holds that

$$
\begin{aligned}
\liminf _{N \rightarrow \infty} \frac{1}{N+1} \#\{0 \leq k \leq N: & \sup _{s \in K_{1}}\left|\varphi_{h}(s+i k h)-f_{1}(s)\right|<\varepsilon, \\
& \left.\sup _{s \in K_{2}}\left|\zeta(s+i k h, \alpha ; \mathfrak{B})-f_{2}(s)\right|<\varepsilon\right\}>0 .
\end{aligned}
$$

Remark 2.2. A typical case when the elements of $L(\mathbb{P}, \alpha, h)$ are linearly independent is that $\alpha$ and $\exp \left\{\frac{2 \pi}{h}\right\}$ are algebraically independent over $\mathbb{Q}$ (see $[2]$ ). On the other hand, in Theorem 2.1 we assume that $\exp \left\{\frac{2 \pi}{h}\right\}$ is rational. Therefore the arithmetic nature of $h$ in Theorem 2.1 is quite different from that in Theorem 1.2

In the present paper we will prove the following more general result, which gives the mixed joint discrete universality of a $\varphi_{h}$ and a collection of periodic Hurwitz zeta-functions. Let $\mathfrak{B}_{j}=\left\{b_{m j}: m \in \mathbb{N}_{0}\right\}, j=1, \ldots, r$, be periodic sequences of complex numbers with the minimal period $k_{j}$. By $\zeta\left(s, \alpha_{j} ; \mathfrak{B}_{j}\right)$ we denote the corresponding periodic Hurwitz zeta-function, $j=1, \ldots, r$.

Theorem 2.3. Suppose that $\varphi_{h}(s)$ and $\exp \left\{\frac{2 \pi}{h}\right\}$ are as in Theorem 2.1, and the numbers $\alpha_{1}, \ldots, \alpha_{r}$ are algebraically independent (hence transcendental; see [17]) over $\mathbb{Q}$. Let $\varphi(s) \in \widetilde{S}, K_{1}$ be a compact subset of $D\left(\sigma^{*}, 1\right), K_{2 j}$ be compact subset of $D(1 / 2,1)$, $j=1, \ldots, r$, all of them with connected complements, and $f_{1} \in H_{0}^{c}\left(K_{1}\right), f_{2 j} \in H^{c}\left(K_{2 j}\right)$. 
Then, for every $\varepsilon>0$,

$$
\begin{aligned}
& \liminf _{N \rightarrow \infty} \frac{1}{N+1} \#\left\{0 \leq k \leq N: \sup _{s \in K_{1}}\left|\varphi_{h}(s+i k h)-f_{1}(s)\right|<\varepsilon\right. \\
&\left.\sup _{1 \leq j \leq r} \sup _{s \in K_{2 j}}\left|\zeta\left(s+i k h, \alpha_{j} ; \mathfrak{B}_{j}\right)-f_{2 j}(s)\right|<\varepsilon\right\}>0 .
\end{aligned}
$$

The mixed joint universality property for the tuple of different types of zeta-functions (one having an Euler product expression and the other without them) was introduced by Mishou in 2007 (see 16]), and independently by Steuding and Sanders in 2006 (see [19]). They obtained that any two holomorphic functions can be approximated simultaneously by shifts of the Riemann zeta-function $\zeta(s)$ and the Hurwitz zeta-function $\zeta(s, \alpha)$.

Generally speaking, the concept of discrete universality was introduced by Reich studying the Dedekind zeta-functions in 1980 (see [18]). Mixed discrete joint universality theorems for zeta-functions are interesting and complicated objects for the investigation, because an important role is played by arithmetic properties of parameters occurring in the theorems. Until this moment only few papers related to this problem have appeared. In the papers $2,3,11,12$, the discrete mixed joint universality for the collection of periodic zeta-functions or its subclasses are studied. The authors' result in [6], which is stated as Theorem 1.2, gives a generalization of the result in [2]. While the most general result (at this moment) for the function $\varphi(s)$ and collection of periodic Hurwitz zeta-functions is contained in [8].

Note that the first attempt to prove mixed discrete joint universality theorem was made by the first author (see [4]). The proof of universality property there unfortunately contains incompleteness, but this work is the origin of our present investigation. In fact, Theorem 2.1 is a generalization of the "corrected" version of [4], as was mentioned in [6].

The present paper is organized in the following way. Since Theorem 2.1 is clearly a special case of Theorem 2.3, it is enough to prove the latter. We separate its proof into two parts: in Section 3 we prove a discrete functional limit theorem which contains the most part of novelty of this paper, and in Section 4 we study the support of the limit measure and give a proof of Theorem 2.3 .

\section{Functional discrete limit theorem}

For the proof of Theorem 2.3, we adopt the well-known probabilistic method (see, for example, 7, 13]), based on joint limit theorems. In this section, we assume $\varphi_{h}(s) \in \mathcal{M}$, and give a proof of a mixed joint discrete limit theorem in the sense of weakly convergent probability measures in the space of holomorphic functions. 
The function $\varphi(s)$ has only finitely many poles, say $s_{1}(\varphi), \ldots, s_{l}(\varphi)$, and let

$$
D_{\varphi}:=\left\{s \in \mathbb{C}: \sigma>\sigma_{0}, \sigma \neq \Re s_{j}(\varphi), j=1, \ldots, l\right\} .
$$

The poles of $\varphi(s)$ and of $\varphi_{h}(s)$ in the region $\sigma>\sigma_{0}$ exactly coincide, and hence the functions $\varphi_{h}(s)$ and $\varphi_{h}(s+i k h)$ are holomorphic in $D_{\varphi}$. Also, the functions $\zeta\left(s, \alpha_{j} ; \mathfrak{B}_{j}\right)$ and $\zeta\left(s+i k h, \alpha_{j} ; \mathfrak{B}_{j}\right)$ are holomorphic in

$$
D_{\zeta}:= \begin{cases}\{s \in \mathbb{C}: \sigma>1 / 2\} & \text { if } \zeta\left(s, \alpha_{j} ; \mathfrak{B}_{j}\right) \text { is entire for all } j, \\ \{s \in \mathbb{C}: \sigma>1 / 2, \sigma \neq 1\} & \text { if } s=1 \text { is a pole of } \zeta\left(s, \alpha_{j} ; \mathfrak{B}_{j}\right) \text { for some } j\end{cases}
$$

(for the arguments, see [5]).

Put $\underline{s}_{r}=\left(s_{1}, s_{21}, \ldots, s_{2 r}\right) \in \mathbb{C}^{r+1}, \underline{\alpha}=\left(\alpha_{1}, \ldots, \alpha_{r}\right)$ and $\underline{\mathfrak{B}}=\left(\mathfrak{B}_{1}, \ldots, \mathfrak{B}_{r}\right)$. Let $D_{1}$ be an open region of $D_{\varphi}$ and $D_{2}$ an open region of $D_{\zeta}$. Write

$$
\underline{H}_{r}:=H\left(D_{1}\right) \times \underbrace{H\left(D_{2}\right) \times \cdots \times H\left(D_{2}\right)}_{r},
$$

where $H\left(D_{j}\right)$ denotes the space of holomorphic functions defined on $D_{j}, j=1,2$. Let

$$
\underline{Z}_{h r}\left(\underline{s}_{r}\right)=\underline{Z}_{h r}\left(\underline{s}_{r}, \underline{\alpha}, \underline{\mathfrak{B}}\right):=\left(\varphi_{h}\left(s_{1}\right), \zeta\left(s_{21}, \alpha_{1} ; \mathfrak{B}_{1}\right), \ldots, \zeta\left(s_{2 r}, \alpha_{r} ; \mathfrak{B}_{r}\right)\right)
$$

and, on $\left(\underline{H}_{r}, \mathcal{B}\left(\underline{H}_{r}\right)\right)$, define

$$
P_{N h r}(A):=\frac{1}{N+1} \#\left\{0 \leq k \leq N: \underline{Z}_{h r}\left(\underline{s}_{r}+i k h\right) \in A\right\}, \quad A \in \mathcal{B}(\underline{H}),
$$

where $\underline{s}_{r}+i k h:=\left(s_{1}+i k h, s_{21}+i k h, \ldots, s_{2 r}+i k h\right)$.

Let $\gamma$ be the unit circle on the complex plane $\mathbb{C}$, i.e., $\gamma:=\{s \in \mathbb{C}:|s|=1\}$. Define two tori

$$
\Omega_{1 h}:=\prod_{p \in \mathbb{P}_{h}} \gamma_{p h} \quad \text { and } \quad \Omega_{2}:=\prod_{m=0}^{\infty} \gamma_{m}
$$

with $\gamma_{p h}=\gamma$ for all $p \in \mathbb{P}_{h}$ and $\gamma_{m}=\gamma$ for all $m \in \mathbb{N}_{0}$, respectively. Further define

$$
\underline{\Omega}_{h r}:=\Omega_{1 h} \times \Omega_{21} \times \cdots \times \Omega_{2 r},
$$

where $\Omega_{2 j}=\Omega_{2}$ for all $j=1, \ldots, r$. By the Tikhonov theorem, the tori $\Omega_{1 h}$ and $\Omega_{2}$ are compact topological Abelian groups (see Lemma 5.1.5 in [9]) with probability Haar measures $m_{H 1 h}$ and $m_{H 2}$ defined on the spaces $\left(\Omega_{1 h}, \mathcal{B}\left(\Omega_{1 h}\right)\right)$ and $\left(\Omega_{2}, \mathcal{B}\left(\Omega_{2}\right)\right)$, respectively. Therefore the torus $\underline{\Omega}_{h r}$ is also a compact topological Abelian group, and, on $\left(\underline{\Omega}_{h r}, \mathcal{B}\left(\underline{\Omega}_{h r}\right)\right)$, there exists a probability Haar measure $m_{H r}^{h}$. Here $m_{H r}^{h}:=m_{H 1 h} \times m_{H 21} \times \cdots \times m_{H 2 r}$ with $m_{H 2 j}=m_{H 2}, j=1, \ldots, r$. This leads to the probability space $\left(\underline{\Omega}_{h r}, \mathcal{B}\left(\underline{\Omega}_{h r}\right), m_{H r}^{h}\right)$. For the elements $\omega_{1 h} \in \Omega_{1 h}$ and $\omega_{2 j} \in \Omega_{2 j}$, denote by $\omega_{1 h}(p)$ and $\omega_{2 j}(m)$ the projections to the coordinate spaces $\gamma_{p h}$ for $p \in \mathbb{P}_{h}$, and $\gamma_{m}$ for $m \in \mathbb{N}_{0}$, respectively. 
Let $\underline{\omega}_{h r}:=\left(\omega_{1 h}, \omega_{21}, \ldots, \omega_{2 r}\right) \in \underline{\Omega}_{h r}$. For $s_{1} \in D_{1}$ and $\omega_{1 h} \in \Omega_{1 h}$, define

$$
\varphi_{h}\left(s_{1}, \omega_{1 h}\right):=\sum_{k \in \mathbb{N}_{m}} \frac{c_{k} \omega_{1 h}(k)}{k^{s_{1}}}=\prod_{k \in \mathbb{N}_{h}} \prod_{j=1}^{g(k)}\left(1-\frac{a_{k}^{(j)} \omega_{1 h}\left(p_{k}\right)^{f(j, k)}}{p_{k}^{\left(s_{1}+\alpha+\beta\right) f(j, k)}}\right)^{-1} .
$$

This series, for all $\omega_{1 h} \in \Omega_{1 h}$, converges absolutely for $\Re s_{1}>1$. From the properties of Dirichlet series it follows that this series converges uniformly almost surely on any compact subsets of $D_{1}$. Therefore, $\varphi_{h}\left(s_{1}, \omega_{1 h}\right)$ is an $H\left(D_{1}\right)$-valued random element defined on the probability space $\left(\Omega_{1 h}, \mathcal{B}\left(\Omega_{1 h}\right), m_{H 1 h}\right)$. On $\left(\Omega_{2 j}, \mathcal{B}\left(\Omega_{2 j}\right), m_{H 2 j}\right)$, define

$$
\zeta\left(s_{2 j}, \alpha_{j}, \omega_{2 j} ; \mathfrak{B}_{j}\right):=\sum_{m=0}^{\infty} \frac{b_{m j} \omega_{2 j}(m)}{\left(m+\alpha_{j}\right)^{s_{2 j}}}, \quad s_{2 j} \in D_{2}, j=1, \ldots, r
$$

for $\omega_{2 j} \in \Omega_{2 j}$, which is an $H\left(D_{2}\right)$-valued random element (for the details, see [10]).

Now, on the probability space $\left(\underline{\Omega}_{h r}, \mathcal{B}\left(\underline{\Omega}_{h r}\right), m_{H r}^{h}\right)$, define an $\underline{H}_{r}$-valued random element $\underline{Z}_{h r}\left(\underline{s}_{r}, \underline{\omega}_{h r}\right)$ by the formula

$$
\begin{aligned}
\underline{Z}_{h r}\left(\underline{s}_{r}, \underline{\omega}_{h r}\right) & =\underline{Z}_{h r}\left(\underline{s}_{r}, \underline{\alpha}, \underline{\omega}_{h r} ; \underline{\mathfrak{B}}\right) \\
& :=\left(\varphi_{h}\left(s_{1}, \omega_{1 h}\right), \zeta\left(s_{21}, \alpha_{1}, \omega_{21} ; \mathfrak{B}_{1}\right), \ldots, \zeta\left(s_{2 r}, \alpha_{r}, \omega_{2 r} ; \mathfrak{B}_{r}\right)\right) .
\end{aligned}
$$

Let $P_{\underline{Z} h r}$ denote its distribution, i.e., $P_{\underline{Z} h r}$ is the probability measure on $\left(\underline{H}_{r}, \mathcal{B}\left(\underline{H}_{r}\right)\right)$ defined by

$$
P_{\underline{Z} h r}(A):=m_{H r}^{h}\left\{\underline{\omega}_{h r} \in \underline{\Omega}_{h r}: \underline{Z}_{h r}\left(\underline{s}_{r}, \underline{\omega}_{h r}\right) \in A\right\}, \quad A \in \mathcal{B}\left(\underline{H}_{r}\right) .
$$

The aim of the present section is to show the following mixed discrete joint limit theorem in the sense of weakly convergent probability measures in the space of holomorphic functions.

Theorem 3.1. Let $\varphi_{h}(s) \in \mathcal{M}$. Suppose that, for $h>0$, $\exp \left\{\frac{2 \pi}{h}\right\}$ is a rational number. Let the numbers $\alpha_{1}, \ldots, \alpha_{r}$ be algebraically independent over $\mathbb{Q}$. Then $P_{N h r}$ converges weakly to $P_{\underline{Z} h r}$ as $N \rightarrow \infty$.

The basic structure of the proof of this theorem is similar to those of same type of discrete limit theorems for $\varphi(s)$ (Theorem 4 in [6] and Theorem 5 in [8]). Therefore we skip some standard details, and focus onto the points which are novel and essential in the present proof.

The first necessary lemma is the following discrete joint limit theorem on the torus $\underline{\Omega}_{h r}$. The assumptions of Theorem 3.1 are essentially used in the proof of this lemma. 
Lemma 3.2. Suppose that $\varphi_{h}(s), \exp \left\{\frac{2 \pi}{h}\right\}$ and $\alpha_{j}, j=1, \ldots, r$, are as in Theorem 3.1 . Then the probability measure

$$
\begin{aligned}
& Q_{N h r}(A) \\
:= & \frac{1}{N+1} \#\left\{0 \leq k \leq N:\left(\left(p^{-i k h}: p \in \mathbb{P}_{h}\right),\left(\left(m+\alpha_{j}\right)^{-i k h}: m \in \mathbb{N}_{0}, j=1, \ldots, r\right)\right) \in A\right\},
\end{aligned}
$$

$A \in \mathcal{B}\left(\underline{\Omega}_{h r}\right)$, converges weakly to the Haar measure $m_{H r}^{h}$ as $N \rightarrow \infty$.

Proof. We use the well-known Fourier transform method. The Fourier transform $g_{N h r}$ of the measure $Q_{N h r}$ is defined by the formula

$$
g_{N h r}\left(\underline{k}, \underline{l}_{1}, \ldots, \underline{l}_{r}\right)=\int_{\underline{\Omega}_{h r}}\left(\prod_{p \in \mathbb{P}_{h}} \omega_{1 h}^{k_{p}}(p) \prod_{j=1}^{r} \prod_{m \in \mathbb{N}_{0}} \omega_{2 j}^{l_{m j}}(m)\right) \mathrm{d} Q_{N h r}
$$

where $\underline{k}=\left(k_{p}: p \in \mathbb{P}_{h}\right), \underline{l}_{j}=\left(l_{m j}: m \in \mathbb{N}_{0}\right), j=1, \ldots, r$, with the condition that only a finite number of integers $k_{p}$ and $l_{m j}$ are distinct from zero. Then, from the definition of $Q_{N h r}$, we have

$$
\begin{aligned}
g_{N h r}\left(\underline{k}, \underline{l}_{1}, \ldots, \underline{l}_{r}\right) & =\frac{1}{N+1} \sum_{k=0}^{N} \prod_{p \in \mathbb{P}_{h}} p^{-i k k_{p} h} \prod_{j=1}^{r} \prod_{m \in \mathbb{N}_{0}}\left(m+\alpha_{j}\right)^{-i k l_{m j} h} \\
& =\frac{1}{N+1} \sum_{k=0}^{N} \exp \left\{-i k h\left(\sum_{p \in \mathbb{P}_{h}} k_{p} \log p+\sum_{j=1}^{r} \sum_{m \in \mathbb{N}_{0}} l_{m j} \log \left(m+\alpha_{j}\right)\right)\right\} .
\end{aligned}
$$

We claim that

$$
\exp \left\{-i h\left(\sum_{p \in \mathbb{P}_{h}} k_{p} \log p+\sum_{j=1}^{r} \sum_{m \in \mathbb{N}_{0}} l_{m j} \log \left(m+\alpha_{j}\right)\right)\right\} \neq 1
$$

when $\left(\underline{k}, \underline{l}_{1}, \ldots, \underline{l}_{r}\right) \neq(\underline{0}, \underline{0}, \ldots, \underline{0})$. In fact, if $(3.1)$ is not true, then

$$
-i h\left(\sum_{p \in \mathbb{P}_{h}} k_{p} \log p+\sum_{j=1}^{r} \sum_{m \in \mathbb{N}_{0}} l_{m j} \log \left(m+\alpha_{j}\right)\right)=2 \pi i r
$$

for some integer $r$. Then

$$
\sum_{p \in \mathbb{P}_{h}} k_{p} \log p+\sum_{j=1}^{r} \sum_{m \in \mathbb{N}_{0}} l_{m j} \log \left(m+\alpha_{j}\right)=-\frac{2 \pi r}{h},
$$

and taking the exponentials, we obtain

$$
\prod_{p \in \mathbb{P}_{h}} p^{k_{p}} \prod_{j=1}^{r} \prod_{m \in \mathbb{N}_{0}}\left(m+\alpha_{j}\right)^{l_{m j}}=\exp \left(-\frac{2 \pi r}{h}\right) .
$$


Since the right-hand side is rational by the assumption, the algebraic independence of $\alpha_{1}, \ldots, \alpha_{r}$ implies that all $l_{m j}=0$. Therefore 3.2 reduces to

$$
\prod_{p \in \mathbb{P}_{h}} p^{k_{p}}=\exp \left(-\frac{2 \pi r}{h}\right)
$$

But this is impossible in view of the condition of $\mathbb{P}_{h}$. Therefore 3.1 is valid, and so,

$$
\begin{aligned}
& g_{N h r}\left(\underline{k}, \underline{l_{1}}, \ldots, \underline{l_{r}}\right) \\
= & \frac{1}{N+1} \frac{1-\exp \left\{-i(N+1) h\left(\sum_{p \in \mathbb{P}_{h}} k_{p} \log p+\sum_{j=1}^{r} \sum_{m \in \mathbb{N}_{0}} l_{m j} \log \left(m+\alpha_{j}\right)\right)\right\}}{1-\exp \left\{-i h\left(\sum_{p \in \mathbb{P}_{h}} k_{p} \log p+\sum_{j=1}^{r} \sum_{m \in \mathbb{N}_{0}} l_{m j} \log \left(m+\alpha_{j}\right)\right)\right\}} .
\end{aligned}
$$

On the other hand, if $\left(\underline{k}, \underline{l}_{1}, \ldots, \underline{l}_{r}\right)=(\underline{0}, \underline{0}, \ldots, \underline{0})$, then the left-hand side of $(3.1)$ is clearly equal to 1 . Thus we have

$$
\lim _{N \rightarrow \infty} g_{N h r}\left(\underline{k}, \underline{l}_{1}, \ldots, \underline{l}_{r}\right)= \begin{cases}1 & \text { if }\left(\underline{k}, \underline{l}_{1}, \ldots, \underline{l}_{r}\right)=(\underline{0}, \underline{0}, \ldots, \underline{0}), \\ 0 & \text { otherwise. }\end{cases}
$$

This and the continuity theorem for probability measures on compact groups prove the lemma.

Suppose that $\sigma_{1}^{*}>1 / 2$ is fixed, and let

$$
v_{1}(m, n)=\exp \left\{-\left(\frac{m}{n}\right)^{\sigma_{1}^{*}}\right\} \quad \text { for } m, n \in \mathbb{N}
$$

and

$$
v_{2}\left(m, n, \alpha_{j}\right)=\exp \left\{-\left(\frac{m+\alpha_{j}}{n+\alpha_{j}}\right)^{\sigma_{1}^{*}}\right\} \quad \text { for } m \in \mathbb{N}_{0}, n \in \mathbb{N}, j=1, \ldots, r .
$$

Define, for $n \in \mathbb{N}$ and a fixed $\underline{\widehat{\omega}}_{h r}=\left(\widehat{\omega}_{1 h}, \widehat{\omega}_{21}, \ldots, \widehat{\omega}_{2 r}\right) \in \underline{\Omega}_{h r}$,

$$
\begin{gathered}
\varphi_{h, n}\left(s_{1}\right):=\sum_{k \in \mathbb{N}_{m}} \frac{c_{k} v_{1}(k, n)}{k^{s_{1}}}, \quad \zeta_{n}\left(s_{2 j}, \alpha_{j} ; \mathfrak{B}_{j}\right):=\sum_{m \in \mathbb{N}_{0}} \frac{b_{m j} v_{2}\left(m, n, \alpha_{j}\right)}{\left(m+\alpha_{j}\right)^{s_{2 j}}}, \\
\varphi_{h, n}\left(s_{1}, \widehat{\omega}_{1 h}\right):=\sum_{k \in \mathbb{N}_{m}} \frac{c_{k} \widehat{\omega}_{1 h}(k) v_{1}(k, n)}{k^{s_{1}}}
\end{gathered}
$$

and

$$
\zeta_{n}\left(s_{2 j}, \alpha_{j}, \widehat{\omega}_{2 j} ; \mathfrak{B}_{j}\right):=\sum_{m \in \mathbb{N}_{0}} \frac{b_{m j} \widehat{\omega}_{2 j}(m) v_{2}\left(m, n, \alpha_{j}\right)}{\left(m+\alpha_{j}\right)^{s_{2 j}}}, \quad j=1, \ldots, r .
$$

These series are absolutely convergent when the real parts of the variables are greater than $1 / 2$ (for the comments, see [5]). Now we consider the weak convergence of the measures

$$
P_{N h, n}(A):=\frac{1}{N+1} \#\left\{0 \leq k \leq N: \underline{Z}_{h r, n}\left(\underline{s}_{r}+i k h\right) \in A\right\}
$$


and

$$
\widehat{P}_{N h, n}(A):=\frac{1}{N+1} \#\left\{0 \leq k \leq N: \underline{Z}_{h r, n}\left(\underline{s}_{r}+i k h, \widehat{\omega}_{h}\right) \in A\right\}
$$

for $A \in \mathcal{B}\left(\underline{H}_{r}\right)$, where

$$
\underline{Z}_{h r, n}\left(\underline{s}_{r}\right):=\left(\varphi_{h, n}\left(s_{1}\right), \zeta_{n}\left(s_{21}, \alpha_{1} ; \mathfrak{B}_{1}\right), \ldots, \zeta_{n}\left(s_{2 r}, \alpha_{r} ; \mathfrak{B}_{r}\right)\right)
$$

and

$$
\underline{Z}_{h r, n}\left(\underline{s}_{r}, \underline{\widehat{\omega}}_{h r}\right):=\left(\varphi_{h, n}\left(s_{1}, \widehat{\omega}_{1 h}\right), \zeta_{n}\left(s_{21}, \alpha_{1}, \widehat{\omega}_{21} ; \mathfrak{B}_{1}\right), \ldots, \zeta_{n}\left(s_{2 r}, \alpha_{r}, \widehat{\omega}_{2 r} ; \mathfrak{B}_{r}\right)\right) .
$$

Lemma 3.3. Suppose that the conditions of Theorem 3.1 hold. Then, for all $n, P_{N h, n}$ and $\widehat{P}_{N h, n}$ both converge weakly to the same probability measure, which we denote by $P_{n}$, on $(\underline{H}, \mathcal{B}(\underline{H}))$ as $N \rightarrow \infty$.

Proof. Because of the absolute convergence of the series for $\varphi_{h, n}(s), \varphi_{h, n}\left(s, \widehat{\omega}_{1 h}\right), \zeta_{n}\left(s, \alpha_{j}\right.$; $\left.\mathfrak{B}_{j}\right)$ and $\zeta_{n}\left(s, \alpha_{j}, \widehat{\omega}_{2 j} ; \mathfrak{B}_{j}\right)$, we can use Lemma 3.2 above and Theorem 5.1 in [1], and argue in a way similar to the proof of Lemma 3.2 in [5] (see also Lemma 3 in [8]), to obtain the statement of the lemma.

Now we need to pass from $\underline{Z}_{h r, n}\left(\underline{s}_{r}\right)$ to $\underline{Z}_{h r}\left(\underline{s}_{r}\right)$ and from $\underline{Z}_{h r, n}\left(\underline{s}_{r}, \underline{\omega}_{h r}\right)$ to $\underline{Z}_{h r}\left(\underline{s}_{r}, \underline{\omega}_{h r}\right)$, respectively. This can be done by using the approximation method together with Lemma 3.3 . For this purpose, we introduce a metric on $\underline{H}_{r}$.

It is known that, for any open region $G \subset \mathbb{C}$, there exists a sequence of compact sets $\left\{K_{l}: l \in \mathbb{N}\right\} \subset G$ such that $G=\bigcup_{l=1}^{\infty} K_{l}, K_{l} \subset K_{l+1}$ for all $l \in \mathbb{N}$, and, if $K$ is a compact set, then $K \subset K_{l}$ for some $l \in \mathbb{N}$. For the functions $f(s), g(s) \in H(G)$, let

$$
\varrho_{G}(f, g)=\sum_{l=1}^{\infty} 2^{-l} \frac{\sup _{s \in K_{l}}|f(s)-g(s)|}{1+\sup _{s \in K_{l}}|f(s)-g(s)|} .
$$

Set $\varrho_{1}=\varrho_{D_{1}}$ and $\varrho_{2}=\varrho_{D_{2}}$. For $\underline{f}=\left(f_{1}, f_{21}, \ldots, f_{2 r}\right)$ and $\underline{g}=\left(g_{1}, g_{21}, \ldots, g_{2 r}\right) \in \underline{H}_{r}$,

$$
\underline{\varrho}(\underline{f}, \underline{g})=\max \left\{\varrho_{1}\left(f_{1}, g_{1}\right), \max _{1 \leq j \leq r} \varrho_{2}\left(f_{2 j}, g_{2 j}\right)\right\} .
$$

Then $\underline{\varrho}(\underline{f}, \underline{g})$ is a metric on the space $\underline{H}_{r}$ which induces its topology of uniform convergence on compacta.

Lemma 3.4. Under conditions of Theorem 3.1, the following relations hold:

$$
\lim _{n \rightarrow \infty} \limsup _{N \rightarrow \infty} \frac{1}{N+1} \sum_{k=0}^{N} \underline{\rho}\left(\underline{Z}_{h r}\left(\underline{s}_{r}+i k h\right), \underline{Z}_{h r, n}\left(\underline{s}_{r}+i k h\right)\right)=0
$$

and, for almost all $\underline{\omega}_{h r} \in \underline{\Omega}_{h r}$,

$$
\left.\lim _{n \rightarrow \infty} \limsup _{N \rightarrow \infty} \frac{1}{N+1} \sum_{k=0}^{N} \underline{\rho}_{h r}\left(\underline{z}_{r}+i k h, \omega_{h}\right), \underline{Z}_{h r, n}\left(\underline{s}_{r}+i k h, \underline{\omega}_{h r}\right)\right)=0 .
$$


To prove this lemma, we need some elements from ergodic theory. Let

$$
f_{h r}=\left\{\left(p^{-i h}: p \in \mathbb{P}_{h}\right),\left(\left(m+\alpha_{j}\right)^{-i h}: m \in \mathbb{N}_{0}, j=1, \ldots, r\right)\right\} \in \underline{\Omega}_{h r}
$$

and, on the probability space $\left(\underline{\Omega}_{h r}, \mathcal{B}\left(\underline{\Omega}_{h r}\right), m_{H r}^{h}\right)$, define the measurable measure-preserving transformation $\Phi_{h r}: \underline{\Omega}_{h r} \rightarrow \underline{\Omega}_{h r}$ by the formula $\Phi_{h r}\left(\underline{\omega}_{h r}\right)=f_{h r} \underline{\omega}_{h r}$ for $\underline{\omega}_{h r} \in \underline{\Omega}_{h r}$. Recall that a set $A \in \mathcal{B}\left(\underline{\Omega}_{h r}\right)$ is called invariant with respect to $\Phi_{h r}$ if the sets $A$ and $\Phi_{h r}(A)$ differ only by a set of zero $m_{H r}^{h}$-measure, and the transformation $\Phi_{h r}$ is ergodic if its $\sigma$-field of invariant sets consists only of sets having $m_{H r}^{h}$-measure equal to 0 or 1 .

Lemma 3.5. Suppose that $\alpha$ is transcendental, $h>0$, and $\exp \left\{\frac{2 \pi}{h}\right\}$ is rational. Then the transformation $\Phi_{h r}$ is ergodic.

In the proof of this lemma, again the conditions for $\alpha$ and $\exp \left\{\frac{2 \pi}{h}\right\}$ play the essential role. Therefore we give the full details.

Proof of Lemma 3.5. Let $\chi$ be a non-trivial character of $\Omega_{h}$. In the proof of Lemma 3.2 , we have already known that such characters are given by

$$
\chi\left(\underline{\omega}_{h r}\right)=\prod_{p \in \mathbb{P}_{h}} \omega_{1 h}^{k_{p}}(p) \prod_{j=1}^{r} \prod_{m \in \mathbb{N}_{0}} \omega_{2 j}^{l_{m j}}(m)
$$

with only a finite number of integers $k_{p}$ and $l_{m j}$ distinct from zero, where $\underline{\omega}_{h r}=\left(\omega_{1 h}, \omega_{21}\right.$, $\left.\ldots, \omega_{2 r}\right), \omega_{1 h} \in \Omega_{1 h}, \omega_{2 j} \in \Omega_{2 j}$. Therefore,

$$
\chi\left(f_{h r}\right)=\exp \left\{-i h\left(\sum_{p \in \mathbb{P}_{h}} k_{p} \log p+\sum_{j=1}^{r} \sum_{m \in \mathbb{N}_{0}} l_{m j} \log \left(m+\alpha_{j}\right)\right)\right\} .
$$

As in the proof of Lemma 3.2 (see the equation (3.1)), under the assumptions for $\alpha$ and $\exp \left\{\frac{2 \pi}{h}\right\}$, we have that

$$
\chi\left(f_{h r}\right) \neq 1
$$

for $\left(\underline{k}, \underline{l}_{1}, \ldots, \underline{l}_{r}\right) \neq(\underline{0}, \underline{0}, \ldots, \underline{0})$.

Denote by $\mathbb{I}_{A}$ the indicator function of the set $A$, and by $\widehat{\mathbb{I}}_{A}(\chi)$ its Fourier transformation. Let $A \in \mathcal{B}\left(\underline{\Omega}_{h r}\right)$ be an invariant set of the transformation $\Phi_{h r}$. Then we have that $\mathbb{I}_{A}\left(f_{h r} \underline{\omega}_{h r}\right)=\mathbb{I}_{A}\left(\underline{\omega}_{h r}\right)$ for almost all $\underline{\omega}_{h r} \in \underline{\Omega}_{h r}$. Therefore we obtain that

$$
\begin{aligned}
\widehat{\mathbb{I}}_{A}(\chi) & =\int_{\underline{\Omega}_{h r}} \chi\left(\underline{\omega}_{h r}\right) \mathbb{I}_{A}\left(\underline{\omega}_{h r}\right) m_{H r}^{h}\left(d \underline{\omega}_{h r}\right)=\int_{\underline{\Omega}_{h r}} \chi\left(f_{h r} \underline{\omega}_{h r}\right) \mathbb{I}_{A}\left(f_{h r} \underline{\omega}_{h r}\right) m_{H r}^{h}\left(d \underline{\omega}_{h r}\right) \\
& =\chi\left(f_{h r}\right) \int_{\underline{\Omega}_{h r}} \chi\left(\underline{\omega}_{h r}\right) \mathbb{I}_{A}\left(\underline{\omega}_{h r}\right) m_{H r}^{h}\left(d \underline{\omega}_{h r}\right)=\chi\left(f_{h r}\right) \widehat{\mathbb{I}}_{A}(\chi) .
\end{aligned}
$$

Hence, in view of 3.3 , for the non-trivial character $\chi$, we have $\widehat{\mathbb{I}}_{A}(\chi)=0$. 
Now let $\chi_{0}$ be the trivial character of $\underline{\Omega}_{h r}$, i.e., $\chi_{0}\left(\underline{\omega}_{h r}\right)=1$ for all $\underline{\omega}_{h r} \in \underline{\Omega}_{h r}$. Put $u_{A}=\widehat{\mathbb{I}}_{A}\left(\chi_{0}\right)$. Taking into account the equalities

$$
\int_{\underline{\Omega}_{h r}} \chi\left(\underline{\omega}_{h r}\right) m_{H r}^{h}\left(d \underline{\omega}_{h r}\right)= \begin{cases}1 & \text { if } \chi=\chi_{0}, \\ 0 & \text { if } \chi \neq \chi_{0},\end{cases}
$$

and the fact $\widehat{\mathbb{I}}_{A}(\chi)=0$ for $\chi \neq \chi_{0}$, obviously we obtain that

$$
\widehat{\mathbb{I}}_{A}(\chi)=u_{A} \int_{\underline{\Omega}_{h r}} \chi\left(\underline{\omega}_{h r}\right) m_{H r}^{h}\left(d \underline{\omega}_{h r}\right)
$$

for every character $\chi$ of $\underline{\Omega}_{h r}$. However the right-hand side is equal to $\widehat{u}_{A}(\chi)$ (here, we regard $u_{A}$ as a constant function on $\underline{\Omega}_{h r}$ ).

Since the function $\mathbb{I}_{A}\left(\omega_{h}\right)$ is determined by its Fourier transform $\widehat{\mathbb{I}}_{A}(\chi)$, it follows that

$$
\mathbb{I}_{A}\left(\underline{\omega}_{h r}\right)=u_{A}
$$

for almost all $\underline{\omega}_{h r} \in \underline{\Omega}_{h r}$. This especially implies $u_{A}=0$ or $u_{A}=1$, because $\mathbb{I}_{A}\left(\underline{\omega}_{h r}\right)$ only takes the values 0 and 1 . Thus from $(3.4)$ it follows that $\mathbb{I}_{A}\left(\underline{\omega}_{h r}\right)=0$ or $\mathbb{I}_{A}\left(\underline{\omega}_{h r}\right)=1$ for almost all $\underline{\omega}_{h r} \in \underline{\Omega}_{h r}$. From this we find that $m_{H r}^{h}(A)=0$ or $m_{H r}^{h}(A)=1$, and, therefore, the transformation $\Phi_{h r}$ is ergodic.

Proof of Lemma 3.4. Since the properties of the function $\varphi_{h}(s)$ are similar to those of $\varphi(s)$, in view of Lemma 3.5, the proof of the lemma goes in the same way as in the proof of Lemma 3 in [6].

On $\left(\underline{H}_{r}, \mathcal{B}\left(\underline{H}_{r}\right)\right)$, for $A \in \mathcal{B}\left(\underline{H}_{r}\right)$, define one more probability measure $\widehat{P}_{N h r}$ by

$$
\widehat{P}_{N h r}(A):=\frac{1}{N+1} \#\left\{0 \leq k \leq N: \underline{Z}_{h r}\left(\underline{s}_{r}+i k h, \underline{\omega}_{h r}\right) \in A\right\} .
$$

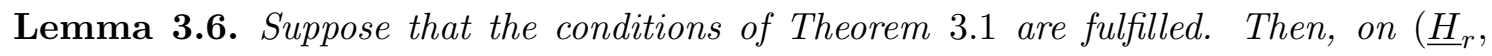
$\left.\mathcal{B}\left(\underline{H}_{r}\right)\right)$, there exists a probability measure $P_{h r}$ such that the measures $P_{N h r}$ and $\widehat{P}_{N h r}$ both converge weakly to $P_{h r}$ as $N \rightarrow \infty$.

Proof. The lemma is an analogue of Lemma 4 in [6], and can be proved similarly as Lemma 5 in 2 .

Proof of Theorem 3.1. Taking into account Lemma 3.6, the proof of Theorem 3.1 will be completed if we can show that the limit measure $P_{h r}$ coincides with the measure $P_{\underline{Z} h r}$. We can do this, using Lemma 3.5 together with the Birkhoff-Khintchine ergodicity theorem, in a standard way (for the details, see 9,20$]$ ). 


\section{Proof of Theorem 2.3}

The deduction of Theorem 2.3 from Theorem 3.1 is now rather standard. We first need the explicit form of the support of the measure $P_{\underline{Z} h r}$. Recall that the support of $P_{\underline{Z} h r}$ is a minimal closed set $S \subset \underline{H}_{r}$ such that $P_{Z h r}(S)=1$.

Let $\varphi_{h}(s) \in \widetilde{S}$, and $K_{1}, K_{2 j}, f_{1}(s)$ and $f_{2 j}(s)$ be as in the statement of Theorem 2.3 . We can find a real number $\sigma_{0}$ such that $\sigma^{*}<\sigma_{0}<1$ and a positive number $M>0$, such that $K_{1}$ is included in the open rectangle

$$
D_{M}=\left\{s \in \mathbb{C}: \sigma_{0}<\sigma<1,|t|<M\right\}
$$

Since $\varphi_{h}(s) \in \widetilde{S}$, its pole is at most at $s=1$ (as for the function $\varphi(s)$ ), then we find that $D_{\varphi}=\left\{s \in \mathbb{C}: \sigma>\sigma_{0}, \sigma \neq 1\right\}$. Therefore, $D_{M}$ is an open subset of $D_{\varphi}$. Also we can find $T>0$ such that $K_{2}$ is included in the open rectangle

$$
D_{T}=\{s \in \mathbb{C}: 1 / 2<\sigma<1,|t|<T\}
$$

Now we choose $D_{1}=D_{M}$ and $D_{2}=D_{T}$ in Theorem 3.1. Denote by $S_{\varphi}$ the set of all functions $f \in H\left(D_{M}\right)$ non-vanishing on $D_{M}$, or constantly equivalent to 0 on $D_{M}$. As a special case of Lemma 5.2 in [7] (when all $l(j)=1, j=1, \ldots, r$, i.e., $\lambda=r$ ), we have

Theorem 4.1. Suppose that $\alpha_{1}, \ldots, \alpha_{r}$ are algebraically independent over $\mathbb{Q}$, and $\exp \left\{\frac{2 \pi}{h}\right\}$ is rational. Then the support of the measure $P_{\underline{Z} h r}$ is the set $S_{\underline{Z} h r}=S_{\varphi} \times H^{r}\left(D_{T}\right)$.

To complete the proof of Theorem 2.3, we use the following well-known Mergelyan theorem on the approximation of analytic functions by polynomials (see [15]).

Lemma 4.2. Let $K \subset \mathbb{C}$ be a compact subset with connected complement, and $f(s)$ be a continuous function on $K$ which is analytic in the interior of $K$. Then, for every $\varepsilon>0$, there exists a polynomial $p(s)$ such that

$$
\sup _{s \in K}|f(s)-p(s)|<\varepsilon
$$

Proof of Theorem 2.3. Since the function $f_{1}(s) \neq 0$ on $K_{1}$, by Lemma 4.2, there exists the polynomials $p_{1}(s)$ and $p_{2 j}(s)$ such that

$$
\sup _{s \in K_{1}}\left|f_{1}(s)-e^{p_{1}(s)}\right|<\frac{\varepsilon}{2} \quad \text { and } \sup _{s \in K_{2}}\left|f_{2 j}(s)-p_{2 j}(s)\right|<\frac{\varepsilon}{2}, \quad j=1, \ldots, r .
$$

Next we define the set

$$
G=\left\{\left(g_{1}, g_{21}, \ldots, g_{2 r}\right) \in \underline{H}_{r}: \sup _{s \in K_{1}}\left|g_{1}(s)-e^{p_{1}(s)}\right|<\frac{\varepsilon}{2}, \sup _{1 \leq j \leq r} \sup _{s \in K_{2 j}}\left|g_{2 j}(s)-p_{2 j}(s)\right|<\frac{\varepsilon}{2}\right\},
$$


which is an open subset of the space $\underline{H}_{r}$, and by Theorem 4.1, it is an open neighbourhood of $\left(e^{p_{1}(s)}, p_{21}(s), \ldots, p_{2 r}(s)\right)$ which is an element of the support of $P_{\underline{Z} h r}$. Therefore, $P_{\underline{Z} h r}(G)>0$. Moreover, Theorem 3.1 and an equivalent statement of the weak convergence in terms of open sets (see [1]) together with the definitions of $P_{N h r}$ and $G$ show that

$$
\liminf _{N \rightarrow \infty} \frac{1}{N+1} \#\left\{0 \leq k \leq N: \underline{Z}_{h r}\left(\underline{s}_{r}+i k h\right) \in G\right\} \geq P_{\underline{Z} h r}(G)>0
$$

or

$$
\begin{aligned}
& \liminf _{N \rightarrow \infty} \frac{1}{N+1} \#\left\{0 \leq k \leq N: \sup _{s \in K_{1}}\left|\varphi_{h}(s+i k h)-e^{p_{1}(s)}\right|<\frac{\varepsilon}{2},\right. \\
&\left.\sup _{1 \leq j \leq r} \sup _{s \in K_{2 j}}\left|\zeta\left(s+i k h, \alpha_{j} ; \mathfrak{B}_{j}\right)-p_{2 j}(s)\right|<\frac{\varepsilon}{2}\right\}>0 .
\end{aligned}
$$

From the last inequality and (4.1) we obtain the assertion of Theorem 2.3 .

\section{Final remarks}

We can generalize Theorem 2.3 to the following direction (as in 8$]$ ). Suppose that $\alpha_{j}$ is a real number such that $\alpha_{j} \in(0,1), j=1, \ldots, r$. We construct the collection of periodic Hurwitz zeta-functions $\zeta\left(s, \alpha_{j} ; \mathfrak{B}_{j l}\right)$ by, for each parameter $\alpha_{j}, j=1, \ldots, r$, attaching a collection of periodic sequences $\mathfrak{B}_{j l}=\left\{b_{m j l} \in \mathbb{C}: m \in \mathbb{N}_{0}\right\}, l=1, \ldots, l(j)$, where $l(j)$ is a positive integer.

Because the proof of the following general mixed discrete joint universality result is quite similar to that of Theorem 2.3 , we give only the statement.

Let $\lambda:=l(1)+\cdots+l(r)$, and, for periodic sequence $\mathfrak{B}_{j l}, k_{j l}$ be the minimal period for coefficients $b_{m j l}$ for each $j=1, \ldots, r, l=1, \ldots, l(j)$. Let $k_{j}$ be the least common multiple of periods $k_{j 1}, \ldots, k_{j l(j)}$, and

$$
B_{j}:=\left(\begin{array}{cccc}
b_{1 j 1} & b_{1 j 2} & \cdots & b_{1 j l(j)} \\
b_{2 j 1} & b_{2 j 2} & \cdots & b_{2 j l(j)} \\
\ldots \ldots & \ldots & \ldots & \ldots \\
\cdots & \ldots & \cdots \\
b_{k_{j} j 1} & b_{k_{j} j 2} & \cdots & b_{k_{j} j l(j)}
\end{array}\right), \quad j=1, \ldots, r .
$$

Theorem 5.1. Suppose that $\varphi_{h}(s)$ belongs to the class $\widetilde{S}, \exp \left\{\frac{2 \pi}{h}\right\}$ is rational number for $h>0, \alpha_{1}, \ldots, \alpha_{r}$ are algebraically independent over $\mathbb{Q}$, and $\operatorname{rank} B_{j}=l(j), j=$ $1, \ldots, r$. Let $K_{1}$ be a compact subset of $D\left(\sigma^{*}, 1\right), K_{2 j l}$ be compact subsets of $D(1 / 2,1)$, $l=1, \ldots, l(j)$, all of them with connected complements. Suppose that $f_{1}(s) \in H_{0}^{c}\left(K_{1}\right)$ and 
$f_{2 j l}(s) \in H^{c}\left(K_{2 j l}\right)$. Then, for every $\varepsilon>0$, it holds that

$$
\begin{aligned}
\liminf _{N \rightarrow \infty} \frac{1}{N+1}\{0 \leq k \leq N: & \sup _{s \in K_{1}}\left|\varphi_{h}(s+i k h)-f_{1}(s)\right|<\varepsilon, \\
& \left.\sup _{1 \leq j \leq r} \sup _{1 \leq l \leq l(j)} \sup _{s \in K_{2 j l}}\left|\zeta\left(s+i k h, \alpha_{j} ; \mathfrak{B}_{j l}\right)-f_{2 j l}(s)\right|<\varepsilon\right\}>0 .
\end{aligned}
$$

\section{References}

[1] P. Billingsley, Convergence of Probability Measures, John Willey \& Sons, New York, 1968.

[2] E. Buivydas and A. Laurinčikas, A discrete version of the Mishou theorem, Ramanujan J. 38 (2015), no. 2, 331-347.

[3] — A generalized joint discrete universality theorem for the Riemann and Hurwitz zeta-functions, Lith. Math. J. 55 (2015), no. 2, 193-206.

[4] R. Kačinskaitè, Joint discrete universality of periodic zeta-functions, Integral Transforms Spec. Funct. 22 (2011), no. 8, 593-601.

[5] R. Kačinskaitè and K. Matsumoto, The mixed joint universality for a class of zetafunctions, Math. Nachr. 288 (2015), no. 16, 1900-1909.

[6] _ On mixed joint discrete universality for a class of zeta-functions, in: Analytic and Probabilistic Methods in Number Theory, 51-66, Vilnius Univ. Leidykla, Vilnius, 2017.

[7] _ Remarks on the mixed joint universality for a class of zeta functions, Bull. Aust. Math. Soc. 95 (2017), no. 2, 187-198.

[8] _ On mixed joint discrete universality for a class of zeta-functions: A further generalization, Math. Model. Anal. 25 (2020), no. 4, 569-583.

[9] A. Laurinčikas, Limit Theorems for the Riemann Zeta-function, Mathematics and its Applications 352, Kluwer Academic Publishers Group, Dordrecht, 1996.

[10] - The joint universality for periodic Hurwitz zeta-functions, Analysis (Munich) 26 (2006), no. 3, 419-428.

[11] _ Joint discrete universality for periodic zeta-functions, Quaest. Math. 42 (2019), no. 5, 687-699. 
[12] _ Joint discrete universality for periodic zeta-functions II, Quaest. Math. 43 (2020), no. 12, 1765-1779.

[13] A. Laurinčikas and D. Šiaučiūnas, A mixed joint universality theorem for zetafunctions III, in: Analytic and Probabilistic Methods in Number Theory, 185-195, TEV, Vilnius, 2012.

[14] K. Matsumoto, Value-distribution of zeta-functions, in: Analytic Number Theory (Tokyo, 1988), 178-187, Lecture Notes in Math. 1434, Springer, Berlin, 1990.

[15] S. N. Mergelyan, Uniform approximations to functions of a complex variable, (Russian) Translated from Uspehi Matem. Nauk (N.S.) 7 (1952), no. 2(48), 31-122; Amer. Math. Soc. Translations Ser. 1, 3, Series and Approximation, 294-391, Amer. Math. Soc., Providence, 1962 (first published in 1954 as Translation no. 101).

[16] H. Mishou, The joint value-distribution of the Riemann zeta function and Hurwitz zeta functions, Liet. Mat. Rink. 47 (2007), no. 1, 62-80; reprinted in Lithuanian Math. J. 47 (2007), no. 1, 32-47.

[17] Yu. V. Nesterenko, Modular functions and transcendence questions, Mat. Sb. 187 (1996), no. 9, 65-96; translation in Sb. Math. 187 (1996), no. 9, 1319-1348.

[18] A. Reich, Werteverteilung von Zetafunktionen, Arch. Math. (Basel) 34 (1980), no. 5, $440-451$.

[19] J. Sander and J. Steuding, Joint universality for sums and products of Dirichlet Lfunctions, Analysis (Munich) 26 (2006), no. 3, 295-312.

[20] J. Steuding, Value-distribution of L-functions, Lecture Notes in Mathematics 1877, Springer, Berlin, 2007.

Roma Kačinskaitè

Department of Mathematics and Statistics, Vytautas Magnus University, Vileikos 8, Kaunas LT-44404, Lithuania

E-mail address: roma.kacinskaite@vdu.1t

Kohji Matsumoto

Graduate School of Mathematics, Nagoya University, Chikusa-ku, Nagoya 464-8602, Japan

E-mail address: kohjimat@math.nagoya-u.ac.jp 